Title:

Author(s):

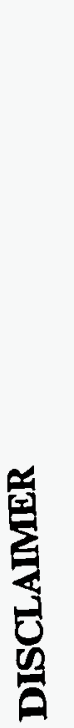

Submitted to:
He ABUNDANCE VARIATIONS IN THE SOLAR WIND: OBSERVATIONS FROM ULYSSES
Bruce L. Barraclough, NIS-1

John T. Gosling, NIS-1

John L. Phillips, NIS-1

David J. McComas, NIS-1

William C. Feldman, NIS-1

Bruce E. Goldstein, JPL

Proceedings of the Eighth International Solar Wind

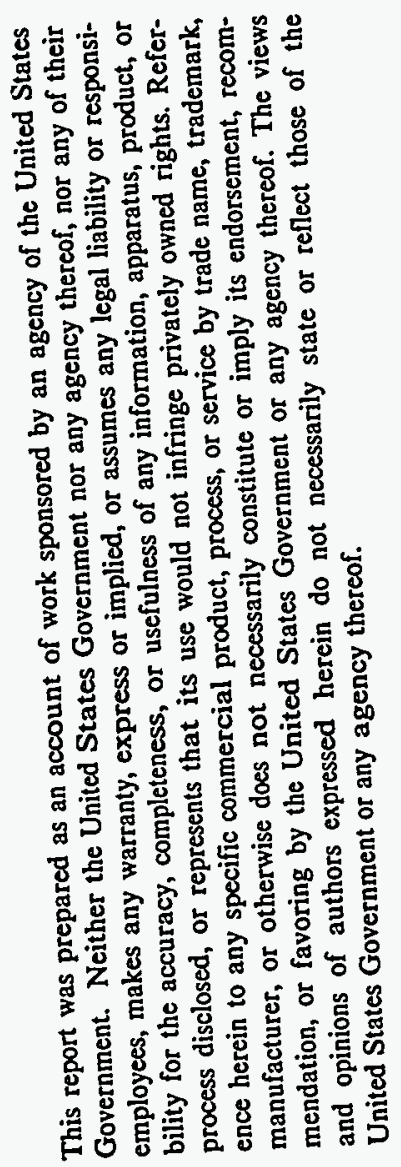

\section{Los Alamos}

NATIONAL LABORATORY

Los Alamos National Laboratory, an affirmative action/equal opportunity empldyer, is operated by the University of California for the U.S. Department of Energy under contract W-7405-ENG-36. By acceptance of this article, the publisher recognizes that the U.S. Government retains a nonexclusive, royalty-free license to publish or reproduce the published form of this contribution, or to allow others to do so, for U.S. Government purposes. The Los Alamos National Laboratory requests that the publisher identify this article as work performed under the auspices of the U.S. Department of Energy. 


\section{DISCLAIMER}

Portions of this document may be illegible in electronic image products. Images are produced from the best available original document. 


\title{
He Abundance Variations in the Solar Wind: Observations from Ulysses
}

\author{
B. L. Barraclough, J. T. Gosling, J. L. Phillips, D. J. McComas and W. C. Feldman \\ Los Alamos National Laboratory, University of California, Los Alamos, NM \\ B.E. Goldstein \\ Jet Propulsion Laboratory, California Institute of Technology, Pasadena, CA
}

\begin{abstract}
$\underline{\text { Abstract }}$
The Ulysses mission is providing the first opportunity to observe variations in solar wind plasma parameters at heliographic latitudes far removed from the ecliptic plane. We present here an overview of the solar wind speed and the variability in helium abundance, $[\mathrm{He}]$, for the entire mission to date, data on [He] in six high latitude coronal mass ejections (CMEs), and a superposed epoch analysis of $[\mathrm{He}]$ variations at the seven heliospheric current sheet (HCS) crossings made during the rapid-latitude-scan portion of the mission. The differences in the variability of the solar wind speed and $[\mathrm{He}]$ in high latitude and equatorial regions are quite striking. Solar wind speed is generally low but highly variable near the solar equator, while at higher latitudes the average speed is quite high (average speed around $760 \mathrm{~km} / \mathrm{s}$ ) with little variability. [He] can vary over nearly two decades at low solar latitudes, while at high latitudes it varies only slightly around an average value of $\sim 4.3 \%$. In contrast to the high [He] that is commonly associated with CMEs observed in the ecliptic, none of the six high-speed CMEs encountered at high southern heliographic latitudes showed any significant variation in helium content from average values. Reasons for this difference between high and low latitude CME observations are not yet understood. A superposed epoch analysis of the [He] during all seven HCS crossings made as Ulysses passed from the southern to northern solar hemisphere shows the expected [He] minimum near the crossing and a broad ( \pm 3 day) region of low $[\mathrm{He}]$ around the crossing time. We discuss how our solar wind [He] observations may provide an accurate measure of the helium composition for the entire convective zone of the Sun.
\end{abstract}




\section{Introduction}

Helium is the second most abundant element in the universe and numerous cosmological models place the probable helium abundance of the primordial nebula that collapsed to form our Sun at about $8-10 \%$ by number (see references in Iben, 1969). The observation that the abundance of helium in the solar wind (defined as $\mathrm{N}_{\mathrm{He}++} / \mathrm{N}_{\mathrm{H}+}$ and denoted hereafter as [He]) averages about $4.3 \%$ and varies over two orders of magnitude has generated numerous theoretical and observational studies which attempt to explain the reasons for the abundance discrepancy and the surprising variability of [He] in the solar wind (see references in Borrini et al., 1982).

The NASA/ESA Ulysses mission is currently providing a unique opportunity to observe the solar wind at previously unexplored heliolatitudes. We report here observations of the abundance and variability of helium in the solar wind for solar latitudes ranging from $\sim 78^{\circ} \mathrm{N}$ to $80.2^{\circ} \mathrm{S}$. The data were obtained from the solar wind plasma ion spectrometer aboard the Ulysses spacecraft (Bame et al., 1992). In brief, the ion instrument is a spherical-section, electrostatic analyzer that provides energy/charge (E/Q) spectra of ambient plasma ions from which the ion distribution function and its derived moments can be obtained. As all ion species in the solar wind are traveling together at approximately the same speed, an E/Q analysis also provides detailed information on the mass/charge distribution of the plasma. The doubly-charged helium peak can normally be well resolved from the much larger proton peak in $\mathrm{E} / \mathrm{Q}$ spectra: $\mathrm{He}^{++}$usually appears at approximately twice the $E / Q$ value of $\mathrm{H}^{+}$. The plasma ion instrument does not provide data on the helium isotope ratios, but a recent paper by Bodmer et al. (1995) gives results on ${ }^{3} \mathrm{He} /{ }^{4} \mathrm{He}$ ratios in the solar wind as seen from Ulysses.

In what follows it should be kept in mind that the observations presented here extend over a period of almost five years, from near solar activity maximum to near solar minimum. Both temporal and spatial (latitudinal) effects are clearly present in the data and we expect to observe a different mix of these effects during Ulysses' next orbit around the Sun, which will occur mostly on the ascending phase of the 11-year solar activity cycle. 
Solar Wind Speed and Helium Abundance Variations as a Function of Heliographic Latitude

As context for the [He] observations, we present in Figure 1 an overview of the solar wind speed for the Ulysses mission from shortly after launch until approach to the northern solar pole. During the early portion of the mission, from launch to several months after Jupiter encounter, it can be seen that the solar wind conditions encountered by Ulysses were widely variable owing to the intermixture of flows from the coronal streamer belt, coronal holes, and numerous coronal mass ejections (CMEs). This is fairly typical for solar maximum conditions.

After the spacecraft was diverted from the ecliptic plane by its encounter with Jupiter in February 1992 and began its climb to high heliographic latitudes, a regular modulation in the solar wind speed began, starting at about $13^{\circ} \mathrm{S}$, as recurrent high-speed plasma streams from the southern polar coronal hole swept across the spacecraft with a roughly 26 day period (Bame et al., 1993). At about $35^{\circ} \mathrm{S}$, the spacecraft finally became continuously immersed in the high-speed wind from the southern polar hole, and speeds reached an average value of approximately $760 \mathrm{~km}$ $\mathrm{s}^{-1}$ and became much less variable (Phillips et al., 1994). The spacecraft then remained in this relatively stable environment for an extended period as it climbed to higher southern latitudes, passed near the south solar pole, and then dropped again to low latitudes. At $22^{\circ} \mathrm{S}$ Ulysses again encountered the highly variable flows typical of equatorial latitudes (Gosling et al., 1995a). After a short equatorial transit, the spacecraft emerged at $21^{\circ} \mathrm{N}$ into the high-speed wind emanating from the northern polar coronal hole where speeds quickly reached values almost identical to those encountered in the high-latitude regions of the southern solar hemisphere (Gosling et al, 1995a).

Figure 1 also shows the solar wind [He] measured during the same time period. During in-ecliptic portions of the mission, [He] was highly variable with values ranging from $0.5 \%$ to near $30 \%$. Most of the observed periods of high [He] can be correlated with transient CMEs passing the spacecraft, while we assume that the periods of very low [He] are predominantly associated with the presence of plasmas from the coronal streamer belt (Borrini et al., 1981). As the spacecraft climbed to high southern latitudes, the variations in [He] became damped quite strongly, with the average value varying little around $4.3 \%$. [He] variability remained remarkably low during the long southern hemisphere transit until the spacecraft again entered the solar equatorial regions in February 1995. Here the variation in [He] again became quite large and 
values often dropped to $<3 \%$, again indicative of streamer belt plasmas. When the spacecraft finally emerged into the high-speed solar wind of the northern polar coronal hole at $\sim 21^{\circ} \mathrm{N}, \mathrm{He}$ abundance variations were dramatically reduced, again closely clustering around the high-speed solar wind average value of $\sim 4.3 \%$.

It is remarkable how constant the [He] was in the high-latitude solar wind during the period of observation: the standard deviation was $\sim 14 \%$ of the average value. This result was anticipated nearly twenty years ago by Bame et al. (1977). They showed that high-speed winds, associated with equatorward extensions of the polar coronal holes, all exhibited fairly uniform [He] and gave an estimate of $4.8 \pm .5 \%$ for the average value. From Figure 1, it is quite evident how widelyvariable helium abundances are when viewed from the perspective of near-ecliptic regions. However, our Ulysses findings point out that low-latitude observations provide only a limited perspective of $[\mathrm{He}]$ variability; the bulk of the wind flowing into interplanetary space (at least during the period of Ulysses' high-latitude observations) is quite uniform in [He] with only the relatively narrow disk of equatorial winds showing high variability.

\section{He Abundance Variations in High-Latitude Coronal Mass Ejections}

During the early phase of the mission (near solar maximum), Ulysses encountered numerous coronal mass ejections (average around two per month), many with very high [He] values. However, for the period from when the spacecraft climbed above about $30^{\circ} \mathrm{S}$ in June 1993 to when it reentered the coronal streamer belt at $22^{\circ} \mathrm{S}$ in February 1995 , only six certain CMEs were encountered (Gosling, 1994a). All six CMEs, which have been identified on the basis of characteristic counterstreaming electron, anomalous field, and low proton temperature signatures, are shown as shaded areas in Figure 2, which also shows [He] data for a five-day period containing each $\mathrm{CME}$.

These high-latitude CMEs are unusual in that a) all were high-speed (average speed $\sim 750$ $\left.\mathrm{km} \mathrm{s}^{-1}\right), \mathrm{b}$ ) half of the six were associated with previously unseen forward-reverse shock pairs (Gosling et al, 1994b), and c) none of the set showed any significant enhancement in [He]. This latter observation is surprising in that high [He] is one of the signatures commonly used to identify CMEs in the ecliptic. The reason for this absence of high [He] may simply be a result of a limited 
statistical sampling, as we have only six certain events with which to work. However, based on in-ecliptic observations, we might reasonably expect at least one or two of the high-latitude CMEs to show significantly elevated [He]. This observation is an unexpected result that awaits further study.

An interesting observation that may bear on the puzzle of the lack of high [He] in highlatitude CMEs is contained in a recent paper by Gosling et al. (1995b). In this study, Ulysses measurements of the fast 26 February $1994 \mathrm{CME}$ shown in Figure 2 are contrasted with the those made by the in-ecliptic IMP 8 spacecraft. It is interesting that while the Ulysses data show only very minor [He] fluctuations (maximum value of $\sim 7 \%$ ) as the CME transited the spacecraft, the IMP data for the same CME show high [He] values ranging up to $21 \%$. Perhaps this CME contained a high $[\mathrm{He}]$ core that was observed at low latitudes while an outer shell with morenormal [He] was the only portion of the CME that was encountered at high latitude, but we are unable to make any firm conclusions as to this scenario.

\section{Helium Abundance Variations during Heliospheric Current Sheet Crossings}

Previous authors have noted that the heliospheric current sheet is typically characterized by minimums in the proton temperature, flow speed and He abundance, and by a maximum in the proton density (Borrini et al, 1981, Gosling et al., 1981). During the rapid-latitude-scan portion of the mission Ulysses made a total of seven HCS crossings (Smith et al., 1995). A superposed epoch plot using one-hour averages of $[\mathrm{He}]$ around these seven $\mathrm{HCS}$ crossings is presented in Figure 3. Here, the zero epoch is the time of the HCS crossing as identified by the Ulysses magnetometer experiment ( $R$. Forsyth, personal communication). It can be seen that a) the $\mathrm{He}$ abundance shows a broad local minimum around the time of the HCS crossing and b) the averaged He abundance for the seven events is quite low for the entire six day period centered on the sector boundary, with the observed [He] never reaching the average solar wind value. Superposed epoch plots of the solar wind flow speed and proton density (not presented here) also show a small minimum in the speed and a small maximum in the proton density, in accord with previous observations. The $[\mathrm{He}]$ and speed minimums and the proton density maximum all show some offset from the nominal HCS crossing time as identified by the Ulysses magnetometer, which may 
be related to an offset between the HCS and the heliospheric plasma sheet as discussed in Winterhalter et al. (1994).

\section{Discussion}

We have presented an overview of the solar wind speed and the magnitude and variation of [He] for the period from just after launch of the Ulysses spacecraft in late 1990 until shortly before the spacecraft attained its maximum northern latitude in July 1995. Figure 1 shows the remarkable lack of variability of $[\mathrm{He}]$ around the observed average value of $4.3 \%$ in the high-speed wind emanating from the polar coronal holes. This lack of variability surprisingly extends even to the six high-speed CMEs that were encountered by Ulysses at high southern latitudes (Figure 2), in stark contrast to what has commonly been observed in the ecliptic. The observation by Gosling et al. (1995b) that one of these six CMEs had a high [He] when it was measured by IMP 8 in the ecliptic plane only serves to deepen this particular puzzle. Finally, we have presented a superposed epoch plot of [He] for the seven HCS crossings made by Ulysses as it passed from the southern to the northern solar hemisphere. Our general finding that there is a broad minimum in [He] during HCS crossings is in accord with previous observations, and reemphasizes the very low abundance of helium that is present in wind emanating from the coronal streamer belt. While we can make the generalizations that a) the quiescent high-speed solar wind appears to have a relatively uniform $[\mathrm{He}]$ of around $4.3 \%$, b) measured $[\mathrm{He}]$ values $>10 \%$ are probably associated only with low-latitude CMEs and c) the lowest [He] values observed are in winds from the coronal streamer belt, the physical reasons for these phenomena remain elusive.

We note that the measured [He] in the high-speed solar wind ( $4.3 \%$ by number) is much less than the cosmic helium abundance ( 8.3 to $10.7 \%$ ) assumed to have constituted the bulk of the primordial Sun (Iben, 1968 and refs. therein). This difference, which has been known for some time (Bame et al., 1977), has been previously explained (e.g. Geiss, 1970) as due to the gravitational settling of $\mathrm{He}$ in the low corona, caused by its large mass/charge ratio. However, there are numerous solar transient phenomena (e.g. coronal bright points, polar plumes, spicules, macrospicules and explosive events) that are observed to be widely active within the coronal holes that cover both solar poles and which should mix low and high altitude coronal material, thereby 
producing time-dependent enhancements of [He] in the solar wind source region. However, we see no evidence for this variability during the long passage of Ulysses through the solar polar regions. This observation can be understood if the entire solar atmosphere is thoroughly mixed by convective transients on a timescale shorter than diffusion can gravitationally settle helium to lower altitudes. If this is so, then the [He] of the high-speed solar wind must provide a faithful measure of the abundance of the entire convective zone of the Sun because there also, convective motions mix the gas faster than diffusion can set up radial gradients (Aller and Chapman, 1960). Further, if the [He] of the convection zone is representative of the entire Sun, as might be expected because of convective mixing during the early Hyashi phase of the Sun (Hyashi, 1966), then models of the helium content of the early solar nebula are in need of revision.

Acknowledgements. The authors wish to thank S. J. Bame for leading the team that built the Ulysses plasma instruments. The work at Los Alamos was performed under the auspices of the U.S. Department of Energy with financial support from NASA.

\section{References}

Aller, L. H. and Chapman, S., Diffusion in the Sun, Ap. J., 1960

Bame, S. J., et al., Evidence for a structure-free state at high solar wind speeds, J. Geophys. Res., 82, 1487, 1977.

Bame, S. J., et al., The Ulysses solar wind plasma experiment, Astron. Astrophys. Suppl.Ser., 92, 237, 1992.

Bame, S. J., et al., Ulysses observations of a recurrent high speed solar wind stream and the heliomagnetic streamer belt, Geophys. Res. Lett., 20, 2323, 1993

Bodmer, R., et al., Solar wind helium isotopic composition from SWICS/Ulysses, Space Sci. Rev., 72, 61, 1995. 
Borrini, G., et al., Solar wind helium and hydrogen structure near the heliospheric current sheet: A signal of coronal streamers at 1 AU, J. Geophys. Res., 86, 4565, 1981.

Borrini, G., et al., Helium abundance enhancements in the solar wind, J. Geophys. Res., 87, $7370,1982$.

Feldman. W. C., et al., Plasma and magnetic fields from the Sun, in The Solar Output and its Variation, O. R. White ed., Colorado Associated University Press, Boulder, 1977.

Feldman, W. C., et al., Long-term variations of selected solar wind properties: $\operatorname{Imp} 6,7$, and 8 results, J. Geophys. Res., 83, 2177, 1978.

Gosling, J. T., et al., Coronal streamers in the solar wind at 1 AU, J. Geophys. Res., 86, 5438, 1981.

Gosling, J. T., Coronal mass ejections in the solar wind at high solar latitudes: An overview, Proc. Third SOHO Workshop, ESA SP-373, 1994a.

Gosling, J. T. , et al., A new class of forward-reverse shock pairs in the solar wind, Geophys. Res. Lett., 21, 2271, $1994 \mathrm{~b}$.

Gosling, J. T., The band of solar wind variability at low heliospheric latitudes near solar activity minimum: Plasma results from the Ulysses rapid latitude scan, Geophys. Res. Lett., in press, $1995 a$

Gosling, J. T., et al., A CME-driven solar wind disturbance observed at both low and high heliographic latitudes, Geophys. Res. Lett., 22, 1753, 1995b

Habbal, S. R., Coronal energy distribution and X-ray activity in the small scale magnetic field of 
the quiet Sun, Ann. Geophysicae, 10, 34, 1992

Hayashi, C., Evolution of protostars, Ann. Rev. Astron. Astrophys., 4, 171, 1966.

Iben, I., The $\mathrm{Cl}^{37}$ solar neutrino experiment and the solar helium abundance, Annals Phys., 54, $164,1969$.

Phillips, J. L., et al., Ulysses at $50^{\circ}$ south: Constant immersion in the high-speed solar wind, Geophys. Res. Lett., 21, 1105, 1994.

Smith, E. J., et al., Results of the Ulysses fast latitude scan: magnetic field observations, Geophys. Res. Lett., submitted, 1995.

Winterhalter, D., et al., The heliospheric plasma sheet, J. Geophys. Res., 99, 6667, 1994.

\section{Figure Captions}

Figure 1 - Overview plot of solar wind speed and [He] as measured by the Los Alamos plasma ion spectrometer aboard Ulysses. Two hour averaged data points are shown. The plot is linear in time with heliographic latitude marked along the top of the figure. Note especially the great difference in variability of the plotted parameters for periods when the spacecraft was near the ecliptic as opposed to when it was at high latitudes.

Figure 2 - Plot showing five days of [He] data around all six high-latitude CMEs observed by Ulysses while in the southern solar hemisphere. Each of the individual plots begins at 00:00 hrs. on the date shown and extends for five days. The shaded area indicates the period when the spacecraft was immersed in the CME. The data points are one hour averages. Note the lack of significant enhancements in [He] for any of these six high-speed CME events.

Figure 3 - Superposed epoch analysis of [He] for all seven HCS crossings made by Ulysses as it 
transited the solar equatorial regions during the rapid-latitude-scan portion of the mission (spacecraft moving rapidly from south to north solar pole). The data shown are one hour averages and the time of the sector boundary crossing is used as the zero epoch. Note the characteristic local minimum in $[\mathrm{He}]$ at the sector boundaries as well as the relatively low [He] during the entire six day period around the crossings. 


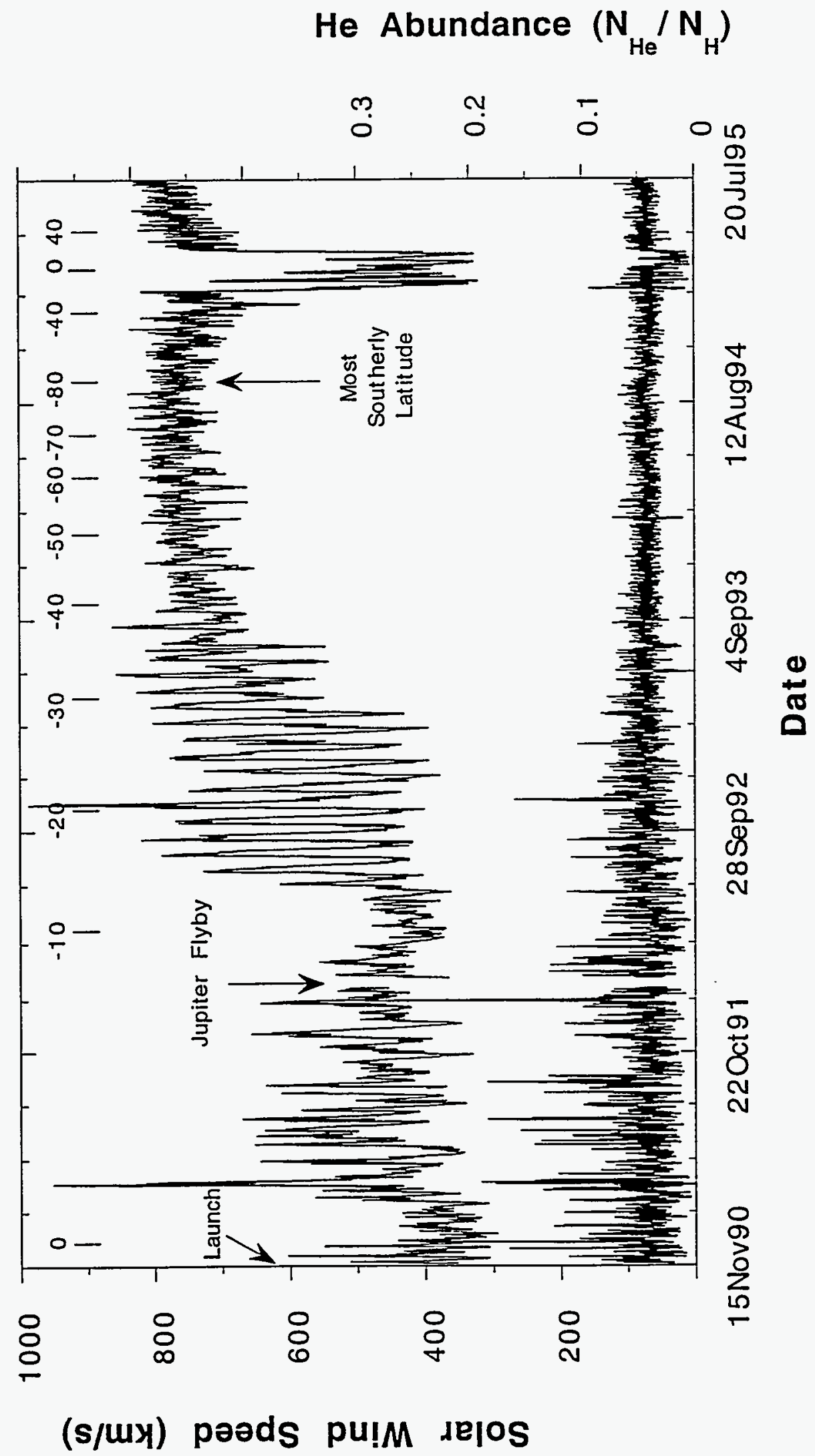




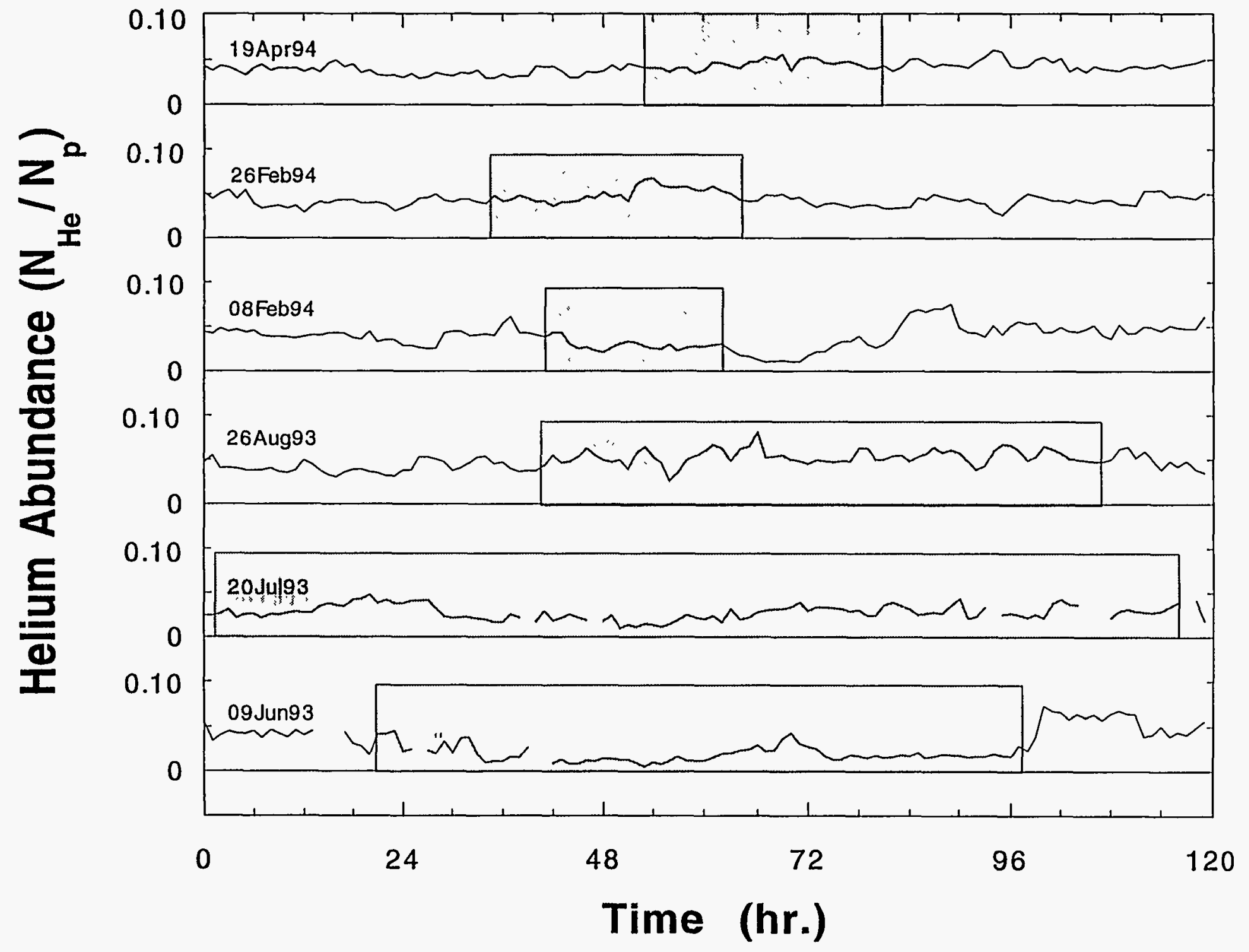




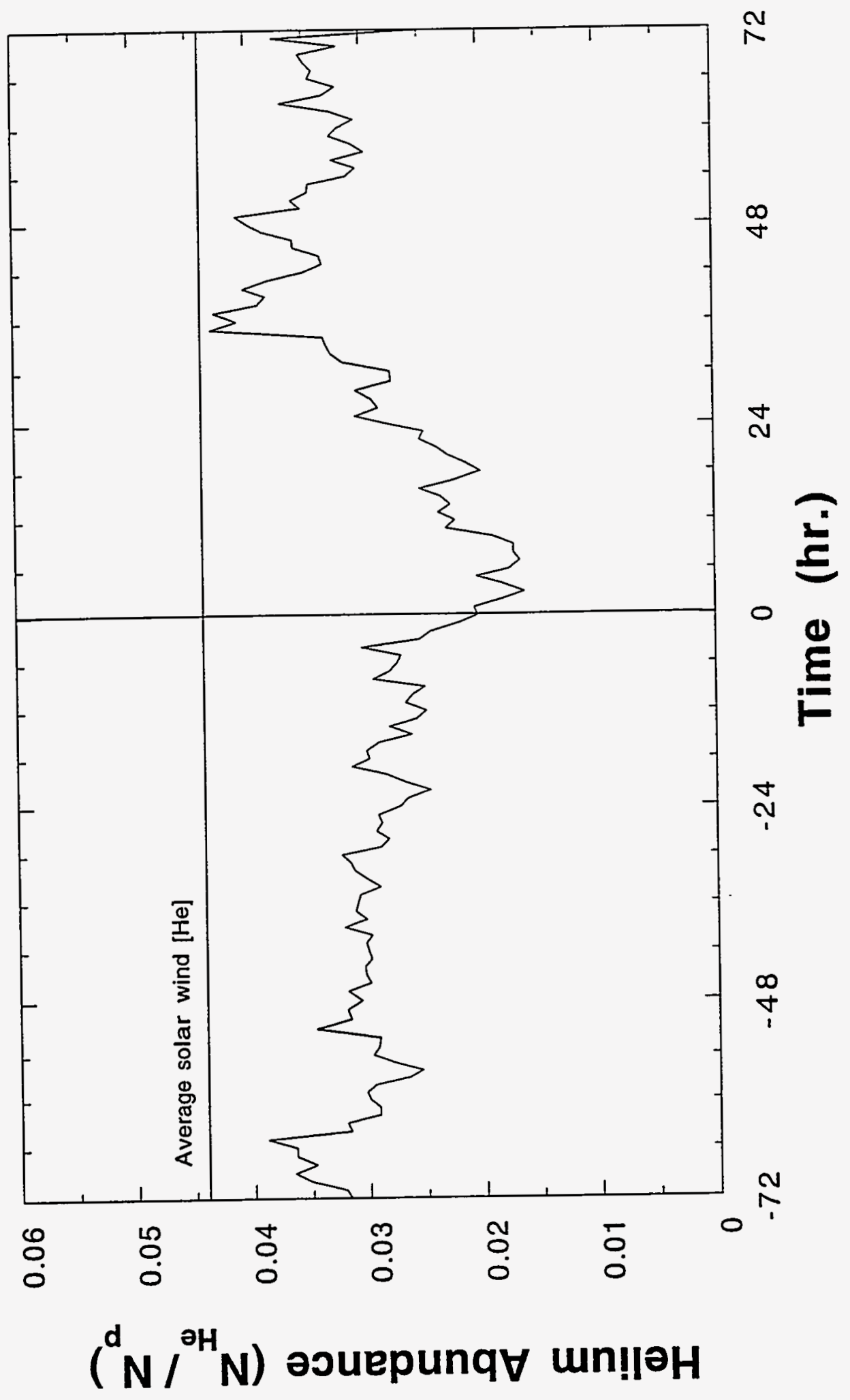

